\title{
HARDY-TYPE ESTIMATES FOR DIRAC OPERATORS
}

\author{
BY JEAN DOLBEAULT \& MARIA J. ESTEBAN, JAVIER \\ DUOANDIKOETXEA \& LUIS VEGA
}

ABSTRACT. - We prove some Hardy type inequalities related to the Dirac operator by elementary methods, for a large class of potentials, which even includes measure valued potentials. Optimality is achieved by the Coulomb potential. When potentials are smooth enough, our estimates provide some spectral information on the operator.

(c) 2007 Elsevier Masson SAS

RÉSUMÉ. - Par des méthodes élémentaires, nous démontrons des inégalités de type Hardy pour des opérateurs de Dirac correspondant à une large classe de potentiels qui comprend des potentiels à valeur mesure. Le cas optimal est réalisé par le potentiel de Coulomb. Pour des potentiels suffisamment réguliers, nos estimations donnent une information sur le spectre de l'opérateur.

(C) 2007 Elsevier Masson SAS

\section{Introduction}

In a recent paper [4], J. Duoandikoetxea and L. Vega proved the following weighted Gagliardo-Nirenberg inequality

$$
\int_{\mathbb{R}^{d}} V(x)|f(x)|^{2} d x \leqslant 2 K[V]\|\nabla f\|_{L^{2}\left(\mathbb{R}^{d}\right)}\|f\|_{L^{2}\left(\mathbb{R}^{d}\right)} \quad \forall f \in H^{1}\left(\mathbb{R}^{d}\right),
$$

for any $d \geqslant 2$ and any nonnegative function $V$. Here the constant $K[V]$ is given by

$$
K[V]:=\inf _{a \in \mathbb{R}^{d}} \sup _{x \in \mathbb{R}^{d}}|x| \int_{0}^{1} V(t x+a) t^{d-1} d t .
$$

J. Duoandikoetxea and L. Vega also proved that the equality holds if $V$ is a multiple of $1 /\left|x-a_{0}\right|$, for some $a_{0} \in \mathbb{R}^{d}$, and in such a case, $f$ has to be a multiple of $e^{-c\left|x-a_{0}\right|}$ with $c>0$ and $K[V]=1 /(d-1)$.

As a consequence, the Schrödinger operator $-\Delta-V$ is semi-bounded from below and satisfies $-\Delta-V \geqslant-K[V]^{2}$ in the sense of operators, since by writing

$$
2 K[V]\|\nabla f\|_{L^{2}\left(\mathbb{R}^{d}\right)}\|f\|_{L^{2}\left(\mathbb{R}^{d}\right)} \leqslant \int_{\mathbb{R}^{d}}|\nabla f|^{2} d x+K[V]^{2} \int_{\mathbb{R}^{d}}|f|^{2} d x,
$$


we obtain

$$
\int_{\mathbb{R}^{d}}|\nabla f|^{2} d x-\int_{\mathbb{R}^{d}} V|f|^{2} d x \geqslant-K[V]^{2} \int_{\mathbb{R}^{d}}|f|^{2} d x \quad \forall f \in H^{1}\left(\mathbb{R}^{d}\right) .
$$

This gives an estimate for the lowest eigenvalue of $-\Delta-V$, and also proves that $-K[V]^{2}$ is an eigenvalue of $-\Delta-V$ if and only if $V$ is a Coulomb potential, i.e., $V(x)=\nu /\left|x-a_{0}\right|$ for some $\nu>0$ and $a_{0} \in \mathbb{R}^{d}$.

The Dirac operator coupled to a potential $V$ takes the form

$$
H_{V}:=-i \alpha \cdot \nabla+\beta-V(x) \mathbb{I}_{4}
$$

where

$$
\alpha:=\left(\begin{array}{cc}
0 & \sigma_{k} \\
\sigma_{k} & 0
\end{array}\right), \quad k=1,2,3, \quad \beta:=\left(\begin{array}{cc}
\mathbb{I}_{2} & 0 \\
0 & -\mathbb{I}_{2}
\end{array}\right)
$$

and $\mathbb{I}_{d}$ is the identity operator on $\mathbb{C}^{d}$. Here $\sigma=\left(\sigma_{k}\right)_{k=1,2,3}$ denotes the family of Pauli matrices:

$$
\sigma_{1}=\left(\begin{array}{cc}
0 & 1 \\
1 & 0
\end{array}\right), \quad \sigma_{2}=\left(\begin{array}{cc}
0 & -i \\
i & 0
\end{array}\right), \quad \sigma_{3}=\left(\begin{array}{cc}
1 & 0 \\
0 & -1
\end{array}\right) .
$$

Such Dirac operators act on four components complex valued spinors defined on $\mathbb{R}^{3}$, i.e., functions of $L^{2}\left(\mathbb{R}^{3}, \mathbb{C}^{4}\right)$. Since the principal part of the operator is homogeneous of degree 1 , Coulomb potentials are critical and eigenvalues are related to some kind of Hardy inequality. This deserves some further explanations.

When $V \equiv 0$, the spectrum of the free Dirac operator $H_{0}$ is $\sigma_{\text {ess }}\left(H_{0}\right):=(-\infty,-1] \cup[1,+\infty)$. If $V(x)=V_{\nu}(x):=\nu /|x|$, it is well known [7] that for any $\nu \in(0,1)$ the Dirac-Coulomb operator $H_{V_{\nu}}$ can be defined as a self-adjoint operator with domain $\mathcal{D}_{\nu}$ satisfying: $H^{1}\left(\mathbb{R}^{3}, \mathbb{C}^{4}\right) \subset$ $\mathcal{D}_{\nu} \subset H^{1 / 2}\left(\mathbb{R}^{3}, \mathbb{C}^{4}\right)$ and spectrum $\sigma_{\text {ess }}\left(H_{0}\right) \cup\left\{\lambda_{1}^{\nu}, \lambda_{2}^{\nu}, \ldots\right\}$ where $\left\{\lambda_{k}^{\nu}\right\}_{k \geqslant 1}$ is the nondecreasing sequence of eigenvalues, all contained in the interval $(0,1)$ and such that $\lambda_{1}^{\nu}=\sqrt{1-\nu^{2}}$, $\lim _{k \rightarrow+\infty} \lambda_{k}^{\nu}=1$, for every $\nu \in(0,1)$. According to [2], for a large set of radial potentials $V$ with singularities not stronger than $1 /|x|$, more precisely, for all those satisfying:

$$
\lim _{|x| \rightarrow+\infty} V(x)=0 \quad \text { and } \quad \frac{\nu}{|x|}+c_{1} \geqslant V \geqslant-c_{2}:=-\sup _{\mathbb{R}^{d}} V,
$$

with $\nu \in(0,1), c_{1}, c_{2} \in \mathbb{R}, c_{1}, c_{2} \geqslant 0, c_{1}+c_{2}-1<\sqrt{1-\nu^{2}}$, if $\lambda_{1}[V]$ is the smallest eigenvalue of the Dirac operator $H_{V}$ in the interval $(-1,1)$, then

$$
\int_{\mathbb{R}^{3}} \frac{|\sigma \cdot \nabla \phi|^{2}}{1+\lambda_{1}[V]+V} d x+\left(1-\lambda_{1}[V]\right) \int_{\mathbb{R}^{3}}|\phi|^{2} d x \geqslant \int_{\mathbb{R}^{3}} V|\phi|^{2} d x \quad \forall \phi \in L^{2}\left(\mathbb{R}^{3}, \mathbb{C}^{2}\right),
$$

with the understanding that some of the terms can be equal to $+\infty$. Although $H_{V}$ is not bounded from below, we shall say in the rest of this paper that $\lambda_{1}[V]$ is the ground state energy level. This can be justified in the non-relativistic limit when physical parameters are taken into account. Asymptotically, one can then relate $\lambda_{1}[V]$ to the lowest eigenvalue of a Schrödinger operator with potential $V$.

Notations in $(*)$ deserve some precisions. We refer to [1] for more details. The spinor $\phi=\left(\begin{array}{l}\phi_{1} \\ \phi_{2}\end{array}\right)$ takes its values in $\mathbb{C}^{2}$ and by $|\phi|^{2},|\nabla \phi|^{2}$ and $|\sigma \cdot \nabla \phi|^{2}$ we denote, respectively, the quantities $\left|\phi_{1}\right|^{2}+\left|\phi_{2}\right|^{2}, \sum_{k=1}^{3}\left(\left|\partial_{k} \phi_{1}\right|^{2}+\left|\partial_{k} \phi_{2}\right|^{2}\right)$ and $\left|\partial_{3} \phi_{1}+\partial_{1} \phi_{2}-i \partial_{2} \phi_{2}\right|^{2}+\left|\partial_{1} \phi_{1}+i \partial_{2} \phi_{1}-\partial_{3} \phi_{2}\right|^{2}$. 
The proof of inequality $(*)$ relies on the following observations. The eigenfunction associated with $\lambda_{1}[V]$ can be characterized as a critical point of the Rayleigh quotient

$$
R[\psi]:=\frac{\left\langle\psi, H_{V} \psi\right\rangle}{\|\psi\|_{L^{2}\left(\mathbb{R}^{3}, \mathbb{C}^{4}\right)}^{2}},
$$

which is obtained by decomposing $\psi=\left(\begin{array}{l}\phi \\ \chi\end{array}\right)$ into an upper component $\phi$ and a lower component $\chi$, where $\phi, \chi \in H^{1}\left(\mathbb{R}^{3}, \mathbb{C}^{2}\right)$ are two components spinors. In a variational context, such a decomposition is known as Talman's decomposition, see [6], and it is proved in [2] that the lowest eigenvalue in $(-1,1)$ is given by

$$
\lambda_{1}[V]=\min _{\phi \neq 0} \max _{\chi} R[\psi] \quad \text { with } \psi=\left(\begin{array}{l}
\phi \\
\chi
\end{array}\right) .
$$

With these notations, the eigenvalue equation becomes a system

$$
\left\{\begin{array}{l}
\mathcal{K} \chi+\phi-V \phi=\lambda_{1}[V] \phi \\
\mathcal{K} \phi-\chi-V \chi=\lambda_{1}[V] \chi
\end{array}\right.
$$

where the operator $\mathcal{K}$ is defined as

$$
\mathcal{K}:=-i \sigma \cdot \nabla .
$$

The method is actually fairly general, see [2] for precisions, and allows for more general decompositions than Talman's decomposition. Moreover, other eigenvalues in the gap can also be characterized under some additional technical conditions: See [3] for a recent result in this direction and for a complete list of references.

We can now rewrite the above characterization of $\lambda_{1}[V]$ as

$$
\lambda_{1}[V]=\min _{\phi \neq 0} \lambda[\phi],
$$

where for any $\phi \in H^{1}\left(\mathbb{R}^{3}, \mathbb{C}^{2}\right)$,

$$
\lambda[\phi]:=\max _{\chi} R[\psi] \quad \text { with } \psi=\left(\begin{array}{l}
\phi \\
\chi
\end{array}\right) .
$$

With the corresponding Euler-Lagrange equation for $\chi$,

$$
\mathcal{K} \phi-\chi-V \chi=\lambda[\phi] \chi,
$$

$\lambda[\phi]$ turns out to be implicitly defined as a solution of

$$
f_{\phi}(\lambda)=0 \quad \text { with } f_{\phi}(\lambda):=\int_{\mathbb{R}^{3}} \frac{|\sigma \cdot \nabla \phi|^{2}}{1+\lambda+V} d x+(1-\lambda) \int_{\mathbb{R}^{3}}|\phi|^{2} d x-\int_{\mathbb{R}^{3}} V|\phi|^{2} d x .
$$

The function $\lambda \mapsto f_{\phi}(\lambda)$ is monotone decreasing with respect to $\lambda$, so $\lambda[\phi]$ is uniquely defined, and for any $\lambda \leqslant \lambda[\phi], f_{\phi}(\lambda) \geqslant 0$. Hence, $f_{\phi}\left(\lambda_{1}[V]\right) \geqslant 0$ for any $\phi \in H^{1}\left(\mathbb{R}^{3}, \mathbb{C}^{2}\right)$, which proves $(*)$. Inequality $(*)$ is achieved by the upper component of the four-spinor of any eigenfunction 
associated with $\lambda_{1}[V]$. In particular if $V=\frac{\nu}{|x|}, \nu \in(0,1)$, we get

$$
\int_{\mathbb{R}^{3}} \frac{|\sigma \cdot \nabla \phi|^{2}}{1+\sqrt{1-\nu^{2}}+\frac{\nu}{|x|}} d x+\left(1-\sqrt{1-\nu^{2}}\right) \int_{\mathbb{R}^{3}}|\phi|^{2} d x \geqslant \nu \int_{\mathbb{R}^{3}} \frac{|\phi|^{2}}{|x|} d x \quad \forall \phi \in L^{2}\left(\mathbb{R}^{3}, \mathbb{C}^{2}\right) .
$$

By taking the limit when $\nu \rightarrow 1$, we get

$$
\int_{\mathbb{R}^{3}} \frac{|\sigma \cdot \nabla \phi|^{2}}{1+\frac{1}{|x|}} d x+\int_{\mathbb{R}^{3}}|\phi|^{2} d x \geqslant \int_{\mathbb{R}^{3}} \frac{|\phi|^{2}}{|x|} d x \quad \forall \phi \in H^{1}\left(\mathbb{R}^{3}, \mathbb{C}^{2}\right)
$$

If we replace $\phi(\cdot)$ by $\varepsilon^{-1} \phi\left(\varepsilon^{-1} \cdot\right)$ and take the limit when $\varepsilon \rightarrow 0$, we obtain

$$
\int_{\mathbb{R}^{3}}|x||\sigma \cdot \nabla \phi|^{2} d x \geqslant \int_{\mathbb{R}^{3}} \frac{|\phi|^{2}}{|x|} d x
$$

By taking $\phi=(g, 0)$ with $g$ purely real, we end up with

$$
\int_{\mathbb{R}^{3}}|x||\nabla g|^{2} d x \geqslant \int_{\mathbb{R}^{3}} \frac{|g|^{2}}{|x|} d x
$$

for all $g \in H^{1}\left(\mathbb{R}^{3}, \mathbb{C}\right)$, which is itself equivalent to

$$
\int_{\mathbb{R}^{3}}|\nabla f|^{2} d x \geqslant \frac{1}{4} \int_{\mathbb{R}^{3}} \frac{|f|^{2}}{|x|^{2}} d x \quad \forall f \in H^{1}\left(\mathbb{R}^{3}, \mathbb{C}\right)
$$

as shown by considering $f=\sqrt{|x|} g$. For more details, see [1,2]. A direct proof and improvements on the potential have even been obtained in [1]. Such results are very similar to the ones known for the Hardy inequality, see [5] for some recent result and further references, or equivalently for the lower estimates for the eigenvalues of Schrödinger operators with critical potentials, i.e., inverse square singular potentials.

Our main result is twofold. We prove a lower bound $\Lambda[V]$ for $\lambda_{1}[V]$ written in the spirit of the approach developed in [4], see Theorem 2. Because of the monotonicity of the function $f_{\phi}$, this proves an inequality like $(*)$ with $\lambda_{1}[V]$ replaced by $\Lambda[V]$ :

$$
\int_{\mathbb{R}^{3}} \frac{|\sigma \cdot \nabla \phi|^{2}}{1+\Lambda[V]+V} d x+(1-\Lambda[V]) \int_{\mathbb{R}^{3}}|\phi|^{2} d x \geqslant \int_{\mathbb{R}^{3}} V|\phi|^{2} d x \quad \forall \phi \in L^{2}\left(\mathbb{R}^{3}, \mathbb{C}^{2}\right)
$$

and, under some technical assumptions, we also show that the equality case is achieved only if $V$ is a Coulomb potential. In such a case $\Lambda[V]=\lambda_{1}[V]$ : See Theorem 1 below.

Section 3 is devoted to the proof of Theorems 1 and 2, which are extended to measure valued potentials in the last section of this paper.

$4^{\text {e }}$ SÉRIE - TOME $40-2007-\mathrm{N}^{\circ} 6$ 


\section{Definitions and main results}

Define the admissible class of radial potentials $\mathcal{A}_{\text {rad }}$ by

$$
V \in \mathcal{A}_{\mathrm{rad}} \Longleftrightarrow\left\{\begin{array}{l}
V: \mathbb{R}^{3} \rightarrow \mathbb{R}^{+} \text {is a nonnegative radial measurable function, } \\
A_{+}[V]:=\sup _{r>0}\left(\frac{1}{r^{2}} \int_{0}^{r} V(t) t^{2} d t\right) \leqslant \frac{1}{2} \\
A_{-}[V]:=\sup _{r>0}\left(r^{2} \int_{r}^{\infty} V(t) \frac{d t}{t^{2}}\right) \leqslant \frac{1}{2}
\end{array}\right.
$$

With a standard abuse of notations, we have written $V(x)=V(|x|)$. Let

$$
\Lambda[V]:=\min _{ \pm} \lambda_{ \pm}, \quad \lambda_{ \pm}:=\sqrt{1-4 A_{ \pm}[V]^{2}}
$$

The fundamental example is the Coulomb potential $V(x)=\frac{\nu}{|x|}$ for which

$$
A_{ \pm}=\frac{\nu}{2} \quad \text { and } \quad \Lambda[V]=\sqrt{1-\nu^{2}}
$$

Remark. - (a) Note that for any $V \in \mathcal{A}_{\text {rad }}, \Lambda[V] \in[0,1]$. Moreover, $V \not \equiv 0$ implies $\Lambda[V]<1$ and $\Lambda[V]=0$ only if $A_{ \pm}[V]=1 / 2$.

(b) If $V \in \mathcal{A}_{\text {rad }}$, then $\widetilde{V}(x)=|x|^{-2} V\left(|x|^{-1}\right)$ is also in $\mathcal{A}_{\text {rad }}$ and $\Lambda[V]=\Lambda[\widetilde{V}]$. This is a simple consequence of $A_{+}[V]=A_{-}[\widetilde{V}]$ and $A_{-}[V]=A_{+}[\widetilde{V}]$. Note that for the Coulomb potential $\widetilde{V}=V$.

With the above notations, we can state our main results.

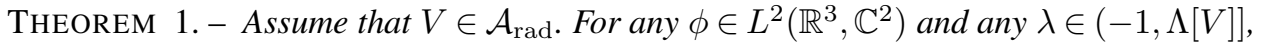

$$
\int_{\mathbb{R}^{3}} V|\phi|^{2} d x \leqslant \int_{\mathbb{R}^{3}} \frac{|\sigma \cdot \nabla \phi|^{2}}{1+\lambda+V} d x+(1-\lambda) \int_{\mathbb{R}^{3}}|\phi|^{2} d x .
$$

Moreover, if $A_{ \pm}<\frac{1}{2}$ and $V \not \equiv 0$, then there exists a non-trivial function $\phi \in L^{2}\left(\mathbb{R}^{3}, \mathbb{C}^{2}\right)$ such that (1) holds as an equality with $\lambda=\Lambda[V]$ if and only if $V$ is the Coulomb potential $V(x)=\frac{\nu}{|x|}$ with $\nu \in(0,1), \nu=\sqrt{1-\Lambda[V]^{2}}$ and $\phi$ is a radial function such that $\phi=B r^{\sqrt{1-\nu^{2}}-1} e^{-\nu r}$ for some constant $B \in \mathbb{C}^{2}$.

Without further restrictions on $\phi$, both sides of the inequality can be infinite. As already explained in the introduction, the motivation for Hardy-type estimates like (1) comes from the Dirac operator. The goal is to give a lower estimate for the ground state level and one can prove the following result.

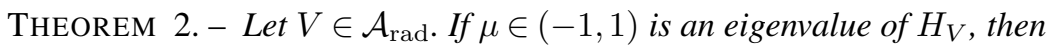

$$
\mu \geqslant \Lambda[V]
$$

As a consequence, if $V \in \mathcal{A}_{\mathrm{rad}}, H_{V}$ has only nonnegative eigenvalues in $(-1,1)$ and $\Lambda[V]$ is a lower bound for all of them. 
Actually, if $V$ is not too singular in order that $H_{V}$ can be defined as a self-adjoint operator with a domain contained in $H^{1 / 2}\left(\mathbb{R}^{3}\right)$, it was proved in [5] that if the maximum of all $\lambda$ such that (1) holds true is in the interval $(-1,1)$, then it is the smallest eigenvalue of $H_{V}$ in $(-1,1)$.

Remark. - (a) If $V \in \mathcal{A}_{\text {rad }}$ and $0 \leqslant W(x) \leqslant V(x)$ a.e., then (1) holds with $W$ instead of $V$ and $\lambda \in(-1, \Lambda[V]]$. Note that the left-hand side of (1) decreases and the right-hand side increases when $V$ is replaced by $W$. Note also that $W$ does not need to be radial.

(b) If $V_{a}(x)=V(a+x)$ is in $\mathcal{A}_{\text {rad }}$ for some $a \in \mathbb{R}^{3}$, then (1) holds for $\lambda \in\left(-1, \Lambda\left[V_{a}\right]\right]$.

Given a general potential $W$ we can combine both remarks and proceed as follows. Consider the radial potentials defined by the least radial majorants of the translates of $W$, that is

$$
W_{a}^{*}(r):=\sup _{\omega \in S^{2}} W(a+r \omega) \text { for } r \geqslant 0 .
$$

If $W_{a}^{*}$ is in $\mathcal{A}_{\text {rad }}$ for some $a \in \mathbb{R}^{3}$, define

$$
\Lambda^{*}[W]:=\sup \left\{\Lambda\left[W_{a}^{*}\right]: a \in \mathbb{R}^{3}, A_{ \pm}\left[W_{a}^{*}\right] \leqslant 1 / 2\right\} .
$$

The following corollary is a simple consequence of Theorem 1 and the preceding remarks.

Corollary 3. - Assume that $W_{a}^{*}$ is in $\mathcal{A}_{\text {rad }}$ for some $a \in \mathbb{R}^{3}$. For any $\phi \in L^{2}\left(\mathbb{R}^{3}, \mathbb{C}^{2}\right)$ and any $\lambda \in\left(-1, \Lambda^{*}[W]\right]$,

$$
\int_{\mathbb{R}^{3}} W|\phi|^{2} d x \leqslant \int_{\mathbb{R}^{3}} \frac{|\sigma \cdot \nabla \phi|^{2}}{1+\lambda+W} d x+(1-\lambda) \int_{\mathbb{R}^{3}}|\phi|^{2} d x,
$$

and $\Lambda^{*}[W]$ is a lower bound for all eigenvalues of $H_{W}$ in the gap $(-1,1)$.

Note that as a special case corresponding to $\lambda=\Lambda[V]$,

$$
\int_{\mathbb{R}^{3}} V|\phi|^{2} d x \leqslant \int_{\mathbb{R}^{3}} \frac{|\sigma \cdot \nabla \phi|^{2}}{1+\Lambda[V]+V} d x+(1-\Lambda[V]) \int_{\mathbb{R}^{3}}|\phi|^{2} d x \quad \forall \phi \in L^{2}\left(\mathbb{R}^{3}, \mathbb{C}^{2}\right) .
$$

Such an inequality is the generalization to Dirac operators of the Hardy inequality for Schrödinger operators established in [4].

\section{Proof of Theorems 1 and 2}

\subsection{Preliminary results}

The Pauli matrices are Hermitian and satisfy the following properties:

$$
\sigma_{j} \sigma_{k}+\sigma_{k} \sigma_{j}=2 \delta_{j k} \mathbb{I}_{2} \quad \forall j, k=1,2,3 .
$$

With a standard abuse of notations, each time a scalar $\delta$ appears in an identity involving operators acting on two-spinors, it has to be understood as $\delta \mathbb{I}_{2}$, where $\mathbb{I}_{2}$ is the identity operator on $\mathbb{C}^{2}$. For any $a, b \in \mathbb{C}^{3}$, we have

$$
(\sigma \cdot a)(\sigma \cdot b)=a \cdot b+i \sigma \cdot(a \times b) .
$$

Applying this formula to $a=x$ and $b=\nabla$, we obtain the following result.

$4^{\mathrm{e}}$ SÉRIE - TOME $40-2007-\mathrm{N}^{\circ} 6$ 
LEMMA 4. - The following equality holds:

$$
\frac{x}{|x|} \cdot \nabla=\left(\sigma \cdot \frac{x}{|x|}\right)(\sigma \cdot \nabla)+\frac{1}{|x|}(\sigma \cdot L) \quad \forall x \in \mathbb{R}^{3},
$$

where $L:=-i x \times \nabla$ is the orbital angular momentum operator.

The next result is concerned with the spectrum of the operator $\sigma \cdot L$. We shall denote by $X_{k}$ the spectral space of $\sigma \cdot L$ associated to the eigenvalue $k$, and by $P_{k}$ the corresponding projector in $L^{2}\left(\mathbb{R}^{3}, \mathbb{C}^{2}\right)$.

Lemma 5. - The spectrum of $\sigma \cdot L$ is the discrete set $\{k \in \mathbb{Z}: k \neq-1\}$ and $\operatorname{Ker}(\sigma \cdot L)$ contains all radial functions. Moreover, if $\phi$ is a continuous function, then $P_{k} \phi(0)=0$ for any $k \in \mathbb{Z} \backslash\{0,-1\}$.

Proof. - The spectrum of the operator $1+\sigma \cdot L$ is the discrete set $\{ \pm 1, \pm 2, \ldots\}$, see [7]. This can be seen by noticing that

$$
1+\sigma \cdot L=J^{2}-L^{2}+\frac{1}{4}, \quad J:=L+\frac{\sigma}{2} .
$$

Then, the fact that the spectrum of $J^{2}$ (resp. $L^{2}$ ) is the set $\left\{j(j+1): j=\frac{1}{2}, \frac{3}{2}, \ldots\right\}$ (resp. $\left\{\ell(\ell+1): \ell=j \pm \frac{1}{2}, j=\frac{1}{2}, \frac{3}{2}, \ldots\right\}$ proves the statement on the spectrum of $1+\sigma \cdot L$.

For all $k \neq 0,-1, P_{k} \phi$ is a linear combination of functions $\psi_{m}^{k}, m=1, \ldots, m_{k}$ which depend only on the angular variable $\omega$ and not on $|x|$, with coefficients

$$
f_{m}^{k}(r)=\int_{S^{2}} P_{k} \phi(r \omega) \psi_{m}^{k}(\omega) d \omega
$$

which are continuous functions of $r=|x|$. According to [7], Section 4.6.4,

$$
f_{m}^{k}(0)=P_{k} \phi(0) \int_{S^{2}} \psi_{m}^{k} d \omega=0,
$$

which proves that $P_{k} \phi(0)=0$ for $k \neq 0,-1$.

The main points are that $L$ commutes with all radial functions and that 0 is not in the spectrum of $1+\sigma \cdot L$. The following result is adapted from [1] and follows trivially from the fact that $\Delta=(\sigma \cdot \nabla)^{2}$ commutes with $\sigma \cdot L$.

Lemma 6. - For any $k, l \in \operatorname{Spec}(\sigma \cdot L), k \neq l, P_{k}(\sigma \cdot \nabla)^{2} P_{l} \equiv P_{l}(\sigma \cdot \nabla)^{2} P_{k} \equiv 0$ in $H^{1}\left(\mathbb{R}^{3}, \mathbb{C}^{2}\right)$.

The radial symmetry of the potential can be taken into account as follows.

COROLlaRY 7. - Any function $\phi \in L^{2}\left(\mathbb{R}^{3}, \mathbb{C}^{2}\right)$ can be written $\phi=\sum_{k \in \mathbb{Z}, k \neq-1} \phi_{k}$ with $\phi_{k} \in X_{k}$ and moreover, if $W$ is a radial function,

$$
\begin{aligned}
\int_{\mathbb{R}^{3}} W|\phi|^{2} d x & =\sum_{k \in \mathbb{Z}, k \neq-1} \int_{\mathbb{R}^{3}} W\left|\phi_{k}\right|^{2} d x, \\
\int_{\mathbb{R}^{3}} W|\sigma \cdot \nabla \phi|^{2} d x & =\sum_{k \in \mathbb{Z}, k \neq-1} \int_{\mathbb{R}^{3}} W\left|\sigma \cdot \nabla \phi_{k}\right|^{2} d x .
\end{aligned}
$$




\subsection{Two useful inequalities}

Here we follow the approach of [4] for the Schrödinger operator. Let $\phi \in \mathcal{D}\left(\mathbb{R}^{3}, \mathbb{C}^{2}\right)$ and write

$$
|\phi(x)|^{2}=\Re\left(\int_{r}^{\infty}-2 \overline{\phi(t \omega)}(\omega \cdot \nabla \phi(t \omega)) d t\right)
$$

for $r=|x|, \omega=x / r$. Assume that $W$ is a radially symmetric function.

$$
\begin{aligned}
\int_{\mathbb{R}^{3}} W|\phi|^{2} d x & =\int_{S^{2}} d \omega \int_{0}^{\infty} W(r \omega)|\phi(r \omega)|^{2} r^{2} d r \\
& =-2 \Re\left(\int_{S^{2}} d \omega \int_{0}^{\infty} W(r \omega) r^{2} d r \int_{r}^{\infty} \overline{\phi(t \omega)}(\omega \cdot \nabla \phi(t \omega)) d t\right) \\
& =-2 \Re\left(\int_{S^{2}} d \omega \int_{0}^{\infty} \overline{\phi(t \omega)}\left(\omega \cdot \nabla \phi(t \omega) t^{2}\right) g_{W}(t) d t\right)
\end{aligned}
$$

where

$$
g_{W}(t):=\frac{1}{t^{2}} \int_{0}^{t} W(r) r^{2} d r .
$$

Using Lemma 4, for any $\delta>0, \mu>-1$, and any nonnegative $V$, we obtain

$$
\begin{aligned}
& \left\langle\left(W+\frac{2}{|x|} g_{W} \sigma \cdot L\right) \phi, \phi\right\rangle \\
& \quad=-2 \Re\left(\int_{\mathbb{R}^{3}}\left(\sqrt{\delta(1+\mu+V)} \sigma \cdot \frac{x}{|x|} \bar{\phi}\right)\left(\frac{\sigma \cdot \nabla \phi}{\sqrt{\delta(1+\mu+V)}}\right) g_{W}(|x|) d x\right) \\
& \quad \leqslant\left\|g_{W}\right\|_{L^{\infty}(0, \infty)}\left[\frac{1}{\delta} \int_{\mathbb{R}^{3}} \frac{|\sigma \cdot \nabla \phi|^{2}}{1+\mu+V} d x+\delta \int_{\mathbb{R}^{3}}(1+\mu+V)|\phi|^{2} d x\right] .
\end{aligned}
$$

Here we use the fact that $\left\langle|x|^{-1} g_{W} \sigma \cdot L \phi, \phi\right\rangle$ is real.

A similar estimate holds with the integral in $r$ taken on the interval $(t, \infty)$. Let $\phi \in \mathcal{D}\left(\mathbb{R}^{3}, \mathbb{C}^{2}\right)$ and write

$$
|\phi(x)|^{2}=|\phi(0)|^{2}+2 \Re\left(\int_{0}^{r} \overline{\phi(t \omega)}(\omega \cdot \nabla \phi(t \omega)) d t\right)
$$

for $r=|x|, \omega=x / r$. Assume that $W$ is a radially symmetric function and that $\phi(0)=0$. Then

$$
\begin{aligned}
\int_{\mathbb{R}^{3}} W|\phi|^{2} d x & =\int_{S^{2}} d \omega \int_{0}^{\infty} W(r \omega)|\phi(r \omega)|^{2} r^{2} d r \\
& =2 \Re\left(\int_{S^{2}} d \omega \int_{0}^{\infty} W(r \omega) r^{2} d r \int_{0}^{r} \overline{\phi(t \omega)}(\omega \cdot \nabla \phi(t \omega)) d t\right)
\end{aligned}
$$

$4^{\mathrm{e}}$ SÉRIE - TOME $40-2007-\mathrm{N}^{\circ} 6$ 


$$
=2 \Re\left(\int_{S^{2}} d \omega \int_{0}^{\infty} \overline{\phi(t \omega)}(\omega \cdot \nabla \phi(t \omega)) t^{2} h_{W}(t) d t\right)
$$

where

$$
h_{W}(t):=\frac{1}{t^{2}} \int_{t}^{\infty} W(r) r^{2} d r
$$

Using Lemma 4, for any $\delta>0, \mu>-1$, and any nonnegative $V$, we obtain

$$
\begin{aligned}
& \left\langle\left(W-\frac{2}{|x|} h_{W} \sigma \cdot L\right) \phi, \phi\right\rangle \\
& \quad=2 \Re\left(\int_{\mathbb{R}^{3}}\left(\sqrt{\delta(1+\mu+V)} \sigma \cdot \frac{x}{|x|} \bar{\phi}\right) d x\left(\frac{\sigma \cdot \nabla \phi}{\sqrt{\delta(1+\mu+V)}}\right) h_{W}(|x|)\right) \\
& \quad \leqslant\left\|h_{W}\right\|_{L^{\infty}(0, \infty)}\left[\frac{1}{\delta} \int_{\mathbb{R}^{3}} \frac{|\sigma \cdot \nabla \phi|^{2}}{1+\mu+V} d x+\delta \int_{\mathbb{R}^{3}}(1+\mu+V)|\phi|^{2} d x\right] .
\end{aligned}
$$

Again we use the fact that $\left\langle|x|^{-1} h_{W} \sigma \cdot L \phi, \phi\right\rangle$ is real.

Summarizing, we have the following result.

LEMMA 8. - With the above notations, for any $\phi \in \mathcal{D}\left(\mathbb{R}^{3}, \mathbb{C}^{2}\right)$, for any $\delta>0, \mu>-1$, and any nonnegative $V$,

$$
\frac{\left\langle\left(W+\frac{2}{|x|} g_{W} \sigma \cdot L\right) \phi, \phi\right\rangle}{\left\|g_{W}\right\|_{L^{\infty}(0, \infty)}} \leqslant \frac{1}{\delta} \int_{\mathbb{R}^{3}} \frac{|\sigma \cdot \nabla \phi|^{2}}{1+\mu+V} d x+\delta \int_{\mathbb{R}^{3}}(1+\mu+V)|\phi|^{2} d x
$$

and, assuming $\phi(0)=0$,

$$
\frac{\left\langle\left(W-\frac{2}{|x|} h_{W} \sigma \cdot L\right) \phi, \phi\right\rangle}{\left\|h_{W}\right\|_{L^{\infty}(0, \infty)}} \leqslant \frac{1}{\delta} \int_{\mathbb{R}^{3}} \frac{|\sigma \cdot \nabla \phi|^{2}}{1+\mu+V} d x+\delta \int_{\mathbb{R}^{3}}(1+\mu+V)|\phi|^{2} d x .
$$

\subsection{Spectral decomposition}

For a given $V \in \mathcal{A}_{\text {rad }}$, define

$$
A_{k}:= \begin{cases}\sup _{r>0}\left(\frac{1}{r^{2(k+1)}} \int_{0}^{r} V(s) s^{2(k+1)} d s\right) & \text { if } k \in \mathbb{Z}, k \geqslant 0, \\ \sup _{r>0}\left(\frac{1}{r^{2(k+1)}} \int_{r}^{+\infty} V(s) s^{2(k+1)} d s\right) & \text { if } k \in \mathbb{Z}, k \leqslant-2 .\end{cases}
$$

Since $V$ is nonnegative, observe that $A_{k} \leqslant A_{0}$ for all $k \in \mathbb{Z}, k \geqslant 0$ and that $A_{k} \leqslant A_{-2}$ for all $k \in \mathbb{Z}, k \leqslant-2$. So, if $V$ belongs to $\mathcal{A}_{\text {rad }}, A_{k} \leqslant 1 / 2$ for all $k \in \mathbb{Z}, k \neq-1$. 


\subsubsection{Nonnegative spectrum of $\sigma \cdot L$}

Assume first that $k \in \mathbb{Z}, k \geqslant 0$, and let us solve the equation

$$
W_{k}+\frac{2 k}{r} g_{k}=V \quad \forall r \in(0, \infty)
$$

where $g_{k}:=g_{W_{k}}$ with the notation (2), i.e.,

$$
g_{k}(r):=\frac{1}{r^{2}} \int_{0}^{r} W_{k}(s) s^{2} d s
$$

Since

$$
\left(r^{2 k} \int_{0}^{r} s^{2} W_{k}(s) d s\right)^{\prime}=r^{2(k+1)} W_{k}+2 k r^{2 k-1} \int_{0}^{r} s^{2} W_{k}(s) d s=r^{2(k+1)} V
$$

Equation (6) can be solved by taking

$$
g_{k}(r)=\frac{1}{r^{2(k+1)}} \int_{0}^{r} V(s) s^{2(k+1)} d s
$$

and

$$
W_{k}=V-\frac{2 k}{r} g_{k}
$$

Note that the relation $g_{k}(r)=\frac{1}{r^{2}} \int_{0}^{r} W_{k}(s) s^{2} d s$ amounts to a simple integration by parts, and that

$$
\left\|g_{k}\right\|_{L^{\infty}(0, \infty)}=A_{k}
$$

by definition of $A_{k}$. Collecting the above estimates and using Lemma 8 , for $\phi=\phi_{k} \in \mathcal{D}\left(\mathbb{R}^{3}, \mathbb{C}^{2}\right)$ such that

$$
\sigma \cdot L \phi_{k}=k \phi_{k}, \quad k \in \mathbb{Z}, k \geqslant 0,
$$

we obtain

$$
\begin{aligned}
\int_{\mathbb{R}^{3}} V\left|\phi_{k}\right|^{2} d x & =\left\langle\left(W_{k}+\frac{2}{|x|} g_{k} \sigma \cdot L\right) \phi_{k}, \phi_{k}\right\rangle \\
& \leqslant A_{k}\left[\frac{1}{\delta} \int_{\mathbb{R}^{3}} \frac{\left|\sigma \cdot \nabla \phi_{k}\right|^{2}}{1+\mu+V} d x+\delta \int_{\mathbb{R}^{3}}(1+\mu+V)\left|\phi_{k}\right|^{2} d x\right]
\end{aligned}
$$

which amounts to

$$
\int_{\mathbb{R}^{3}} \frac{\left|\sigma \cdot \nabla \phi_{k}\right|^{2}}{1+\mu+V} d x+(1-\mu) \int_{\mathbb{R}^{3}}\left|\phi_{k}\right|^{2} d x-\int_{\mathbb{R}^{3}} V\left|\phi_{k}\right|^{2} d x \geqslant 0
$$

if $\mu$ and $\delta$ satisfy the equations

$4^{\mathrm{e}}$ SÉRIE - TOME $40-2007-\mathrm{N}^{\circ} 6$ 


$$
\begin{aligned}
& 1-\mu=\delta^{2}(1+\mu), \\
& A_{k} \delta^{2}-\delta+A_{k}=0 .
\end{aligned}
$$

Solutions to the above system of scalar equations are given by

$$
\begin{gathered}
\delta=\delta_{k}^{ \pm}=\frac{1}{2 A_{k}}\left(1 \pm \sqrt{1-4 A_{k}^{2}}\right), \\
\mu=\mu_{k}^{ \pm}=\frac{1-\left|\delta_{k}^{ \pm}\right|^{2}}{1+\left|\delta_{k}^{ \pm}\right|^{2}}=\mp \sqrt{1-4 A_{k}^{2}} .
\end{gathered}
$$

We observe that $\mu_{k}^{+}<0<\mu_{k}^{-}=\sqrt{1-4 A_{k}^{2}}$. We can therefore choose $\delta=\delta_{k}^{-}$and $\mu=\mu_{k}^{-}$in order to maximize $\mu$, and get

$$
\int_{\mathbb{R}^{3}} \frac{\left|\sigma \cdot \nabla \phi_{k}\right|^{2}}{1+\mu_{k}^{-}+V} d x+\left(1-\mu_{k}^{-}\right) \int_{\mathbb{R}^{3}}\left|\phi_{k}\right|^{2} d x-\int_{\mathbb{R}^{3}} V\left|\phi_{k}\right|^{2} d x \geqslant 0
$$

for any $\phi_{k} \in \mathcal{D}\left(\mathbb{R}^{3}, \mathbb{C}^{2}\right)$ such that

$$
\sigma \cdot L \phi_{k}=k \phi_{k}, \quad k \in \mathbb{Z}, k \geqslant 0
$$

\subsubsection{Negative spectrum of $\sigma \cdot L$}

Similarly, for $k \in \mathbb{Z}, k \leqslant-2$, let us solve the equation

$$
W_{k}-\frac{2 k}{r} h_{k}=V \quad \forall r \in(0, \infty)
$$

where $h_{k}:=h_{W_{k}}$ with the notation (3), i.e.,

$$
h_{k}(r):=\frac{1}{r^{2}} \int_{r}^{\infty} W_{k}(s) s^{2} d s
$$

Since

$$
\left(r^{2 k} \int_{r}^{\infty} s^{2} W_{k}(s) d s\right)^{\prime}=-r^{2(k+1)} W_{k}+2 k r^{2 k-1} \int_{r}^{\infty} s^{2} W_{k}(s) d s=-r^{2(k+1)} V,
$$

Equation (7) can be solved by taking

$$
h_{k}(r)=\frac{1}{r^{2(k+1)}} \int_{r}^{\infty} V(s) s^{2(k+1)} d s
$$

and $W_{k}=\frac{2 k}{r} h_{k}+V$. Note that the relation $h_{k}(r)=\frac{1}{r^{2}} \int_{r}^{\infty} W_{k}(s) s^{2} d s$ holds as a consequence of a simple integration by parts, and that

$$
\left\|h_{k}\right\|_{L^{\infty}(0, \infty)}=A_{k}
$$

by definition of $A_{k}$, for any $k \in \mathbb{Z}, k \leqslant-2$.

ANNALES SCIENTIFIQUES DE L'ÉCOLE NORMALE SUPÉRIEURE 
Let $\phi=\phi_{k} \in \mathcal{D}\left(\mathbb{R}^{3}, \mathbb{C}^{2}\right)$ be such that

$$
\sigma \cdot L \phi_{k}=k \phi_{k}, \quad k \in \mathbb{Z}, k \leqslant-2,
$$

and note that $\phi_{k}(0)=0$ by the remark following Lemma 5. Collecting the above estimates, we obtain exactly as in Section 3.3.1

$$
\begin{aligned}
\int_{\mathbb{R}^{3}} V\left|\phi_{k}\right|^{2} d x & =\left\langle\left(W_{k}-\frac{2}{|x|} h_{k} \sigma \cdot L\right) \phi_{k}, \phi_{k}\right\rangle \\
& \leqslant A_{k}\left[\frac{1}{\delta} \int_{\mathbb{R}^{3}} \frac{\left|\sigma \cdot \nabla \phi_{k}\right|^{2}}{1+\mu+V} d x+\delta \int_{\mathbb{R}^{3}}(1+\mu+V)\left|\phi_{k}\right|^{2} d x\right]
\end{aligned}
$$

which amounts to

$$
\int_{\mathbb{R}^{3}} \frac{\left|\sigma \cdot \nabla \phi_{k}\right|^{2}}{1+\mu+V} d x+(1-\mu) \int_{\mathbb{R}^{3}}\left|\phi_{k}\right|^{2} d x-\int_{\mathbb{R}^{3}} V\left|\phi_{k}\right|^{2} d x \geqslant 0
$$

if $\mu$ and $\delta$ satisfy the equations

$$
\begin{aligned}
& 1-\mu=\delta^{2}(1+\mu), \\
& A_{k} \delta^{2}-\delta+A_{k}=0 .
\end{aligned}
$$

The proof goes exactly as in the case of the nonnegative spectrum of $\sigma \cdot L$. Solutions to the above system of scalar equations are given by

$$
\begin{gathered}
\delta=\delta_{k}^{ \pm}=\frac{1}{2 A_{k}}\left(1 \pm \sqrt{1-4 A_{k}^{2}}\right), \\
\mu=\mu_{k}^{ \pm}=\frac{1-\left|\delta_{k}^{ \pm}\right|^{2}}{1+\left|\delta_{k}^{ \pm}\right|^{2}}=\mp \sqrt{1-4 A_{k}^{2}} .
\end{gathered}
$$

We observe that $\mu_{k}^{+}<0<\mu_{k}^{-}=4 A_{k}^{2}$. We can therefore choose $\delta=\delta_{k}^{-}$and $\mu=\mu_{k}^{-}$in order to maximize $\mu$, and get

$$
\int_{\mathbb{R}^{3}} \frac{\left|\sigma \cdot \nabla \phi_{k}\right|^{2}}{1+\mu_{k}^{-}+V} d x+\left(1-\mu_{k}^{-}\right) \int_{\mathbb{R}^{3}}\left|\phi_{k}\right|^{2} d x-\int_{\mathbb{R}^{3}} V\left|\phi_{k}\right|^{2} d x \geqslant 0
$$

for any $\phi_{k}, k \in \mathbb{Z}, k \leqslant-2$, such that

$$
\sigma \cdot L \phi_{k}=k \phi_{k}
$$

\subsubsection{Partial result on the eigenspaces of $\sigma \cdot L$}

Collecting the estimates obtained in Sections 3.3.1 and 3.3.2 for smooth functions, and then using a density argument, we can state the following result.

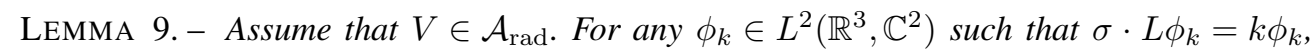
$k \in \mathbb{Z}, k \neq-1$,

$$
\int_{\mathbb{R}^{3}} V\left|\phi_{k}\right|^{2} d x \leqslant \int_{\mathbb{R}^{3}} \frac{\left|\sigma \cdot \nabla \phi_{k}\right|^{2}}{1+\mu_{k}^{-}+V} d x+\left(1-\mu_{k}^{-}\right) \int_{\mathbb{R}^{3}}\left|\phi_{k}\right|^{2} d x .
$$

$4^{\text {e }}$ SÉRIE - TOME $40-2007-\mathrm{N}^{\circ} 6$ 


\subsection{Completion of the proof of Theorem 1}

Inequality (1) with $\lambda=\Lambda[V]$ follows from Corollary 7 and Lemma 9 using $A_{k} \leqslant A_{0}=A_{+}[V]$ for all $k \in \mathbb{Z}, k \geqslant 0$ and $A_{k} \leqslant A_{-2}=A_{-}[V]$ for all $k \in \mathbb{Z}, k \leqslant-2$. The case $\lambda<\Lambda[V]$ is a consequence of the monotonicity with respect to $\lambda$.

With the notations of Section 3.3, if the equality case in (1) with $\lambda=\Lambda[V]$ occurs for some non-trivial function $\phi \in L^{2}\left(\mathbb{R}^{3}, \mathbb{C}^{2}\right)$, then equality occurs in (4) for $W=W_{k}$, for any $k \geqslant 0$, and equality also occurs in (5) for $W=W_{k}$, for any $k \leqslant-2$. If we decompose $\phi$ on the eigenspaces of $\sigma \cdot L$ as $\sum_{k \neq-1} P_{k} \phi$, this means that for any $k_{0} \in \mathbb{Z}, k_{0} \neq-1$, such that $\phi_{k_{0}}:=P_{k_{0}} \phi \neq 0$,

(1) if $k_{0} \geqslant 0$, then $g_{k_{0}} \equiv A_{k_{0}}$ is constant a.e.

(2) if $k_{0} \leqslant-2$, then $h_{k_{0}} \equiv A_{k_{0}}$ is constant a.e.

Hence a derivation with respect to $r$ of $A_{k_{0}} \equiv \frac{1}{r^{2\left(k_{0}+1\right)}} \int_{0}^{r} V(s) s^{2\left(k_{0}+1\right)} d s$ in the first case, and of $A_{k_{0}} \equiv \frac{1}{r^{2\left(k_{0}+1\right)}} \int_{r}^{+\infty} V(s) s^{2\left(k_{0}+1\right)} d s$ in the second case, shows that

$$
V(r)=\frac{\nu}{r} \quad \forall r \in(0, \infty)
$$

with $\nu=2\left|k_{0}+1\right| A_{k_{0}}$. For this potential we compute all $A_{k}$ 's and observe that $A_{0}=A_{-2}=$ $\nu / 2$ and that $A_{k}<\nu / 2$ for all $k \neq-2,0$. So, $P_{k} \phi=0$ for all $k \neq-2,0$. For $k_{0}=0$, $-2, \phi_{k_{0}}=P_{k_{0}} \phi=f_{k_{0}}(r) F_{k_{0}}$, where $F_{k_{0}}$ is an eigenfunction of $\sigma \cdot L$ with eigenvalue $k_{0}$ depending only on the angular variables and not on $|x|$. Equality in (4) and $k_{0}=0$ means that $\left(\sigma \cdot \frac{x}{|x|}\right)(\sigma \cdot \nabla) \phi_{0}=-\delta_{0}^{-}\left(1+\mu_{0}^{-}+V\right) \phi_{0}$, that is, from Lemma $4, f_{0}(r)$ is a solution to the equation

$$
f_{0}^{\prime}=-\delta_{0}^{-}\left(1+\mu_{0}^{-}+\frac{\nu}{r}\right) f_{0}
$$

where $\delta_{0}^{-}\left(1+\mu_{0}^{-}\right)=\nu$ is solved by $\nu \delta_{0}^{-}=1-\sqrt{1-\nu^{2}}$. Solving the equation yields

$$
f_{0}(r)=A r^{-1+\sqrt{1-\nu^{2}}} e^{-\nu r}
$$

for some constant $A \in \mathbb{C}$. On the other hand, equality for (5) and $k_{0}=-2$ means that $\left(\sigma \cdot \frac{x}{|x|}\right)(\sigma \cdot \nabla) \phi_{-2}=\delta_{-2}^{-}\left(1+\mu_{-2}^{-}+V\right) \phi_{-2}$, that is, from Lemma $4, f_{-2}(r)$ is a solution to the equation

$$
f_{-2}^{\prime}=\delta_{-2}^{-}\left(1+\mu_{-2}^{-}+\frac{\nu}{r}\right) f_{-2}-\frac{2}{r} f_{-2}
$$

where $\delta_{-2}^{-}\left(1+\mu_{-2}^{-}\right)=\nu$ is solved by $\nu \delta_{-2}^{-}=1-\sqrt{1-\nu^{2}}$. Solving the equation yields

$$
f_{-2}(r)=A r^{-1-\sqrt{1-\nu^{2}}} e^{\nu r}
$$

for some constant $A \in \mathbb{C}$. Hence $f_{-2}$ is not in $L^{2}\left(\mathbb{R}^{3}\right)$ unless $A=0$. The proof is completed by recalling that the eigenspace of $\sigma \cdot L$ corresponding to the 0 eigenvalue is the space $L^{2}\left(r^{2} d r, \mathbb{C}^{2}\right)$, see [7], Section 4.6.4, while, reciprocally, all computations are explicit in the case $V(r)=\nu / r$.

\subsection{Proof of Theorem 2}

Assume that $\mu \in(-1,1)$ is an eigenvalue of $H_{V}$ and consider an associated eigenfunction $\psi=\left(\begin{array}{l}\phi \\ \chi\end{array}\right) \in L^{2}\left(\mathbb{R}^{3}, \mathbb{C}^{4}\right)$ satisfying $H_{V} \psi=\mu \psi$ or, what is equivalent, solutions $\phi$ and $\chi$ of the 
following system of two equations:

$$
\mathcal{K} \chi+(1-V) \phi=\mu \phi, \quad \mathcal{K} \phi-(1+V) \chi=\mu \chi
$$

Using the second equation, we can eliminate $\chi$ to get

$$
\chi=\frac{\mathcal{K} \phi}{1+\mu+V}, \quad \mathcal{K}\left(\frac{\mathcal{K} \phi}{1+\mu+V}\right)+(1-V) \phi=\mu \phi .
$$

Multiplying the last equation by $\phi$ and integrating over $\mathbb{R}^{3}$, we get

$$
f_{\phi}(\mu):=\int_{\mathbb{R}^{3}} \frac{|\sigma \cdot \nabla \phi|^{2}}{1+\mu+V} d x+(1-\mu) \int_{\mathbb{R}^{3}}|\phi|^{2} d x-\int_{\mathbb{R}^{3}} V|\phi|^{2} d x=0 .
$$

By monotonicity of $f_{\phi}, f_{\phi}(\lambda)<0$ for any $\lambda>\mu$. Hence, by (1), $\Lambda[V] \leqslant \mu$.

\section{Measure valued potentials}

Let $\mathcal{M}_{\mathrm{rad}}^{\text {sing }}$ be the set of nonnegative radial Radon measure with support in zero measure sets, with respect to Lebesgue measure. We can extend the class of admissible radial potentials $\mathcal{A}_{\text {rad }}$ to the larger class $\tilde{\mathcal{A}}_{\text {rad }}$ of measure valued potentials corresponding to

$$
\mathcal{R}=V+\mathcal{S} \in \tilde{\mathcal{A}}_{\mathrm{rad}} \Longleftrightarrow\left\{\begin{array}{l}
V \in \mathcal{A}_{\mathrm{rad}} \text { and } \mathcal{S} \in \mathcal{M}_{\mathrm{rad}}^{\mathrm{sing}} \\
A_{+}[\mathcal{R}]:=\sup _{r>0}\left[\frac{1}{r^{2}}\left(\int_{0}^{r} V(t) t^{2} d t+\int_{0}^{r} t^{2} d \mathcal{S}\right)\right] \leqslant \frac{1}{2} \\
A_{-}[\mathcal{R}]:=\sup _{r>0}\left[r^{2}\left(\int_{r}^{\infty} V(t) \frac{d t}{t^{2}}+\int_{r}^{\infty} t^{-2} d \mathcal{S}\right)\right] \leqslant \frac{1}{2}
\end{array}\right.
$$

where we do for $\mathcal{S}$ the usual abuse of notations of identifying $x$ with $|x|$. With $\delta_{ \pm}, \lambda_{ \pm}$and $\Lambda[\mathcal{R}]$ defined in terms of $A_{ \pm}[\mathcal{R}]$ exactly as in Section 2, we obtain the following result.

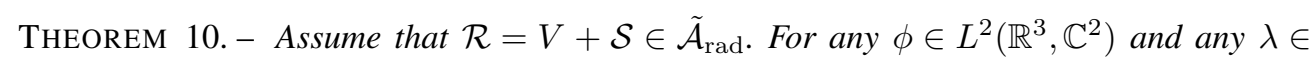
$(-1, \Lambda[\mathcal{R}]]$,

$$
\int_{\mathbb{R}^{3}} V|\phi|^{2} d x+\int_{\mathbb{R}^{3}}|\phi|^{2} d \mathcal{S} \leqslant \int_{\mathbb{R}^{3}} \frac{|\sigma \cdot \nabla \phi|^{2}}{1+\lambda+V} d x+(1-\lambda) \int_{\mathbb{R}^{3}}|\phi|^{2} d x .
$$

Moreover, if $A_{ \pm}<\frac{1}{2}$ and $\Lambda[\mathcal{R}]<1$, then there exists a non-trivial function $\phi \in L^{2}\left(\mathbb{R}^{3}, \mathbb{C}^{2}\right)$ such that (1) holds as an equality with $\lambda=\Lambda[\mathcal{R}]$ if and only if $\mathcal{S}=0$ and $V$ is the Coulomb potential $V(x)=\frac{\nu}{|x|}$, with $\nu \in(0,1)$ and $\nu=\sqrt{1-\Lambda[\mathcal{R}]^{2}}$.

Proof. - Consider a sequence of functions $\left(W_{n}\right)_{n \in \mathbb{N}} \subset \mathcal{A}_{\text {rad }}$ such that $\mathcal{S}=\lim _{n \rightarrow \infty} W_{n}$ vaguely in the sense of measures and $\Lambda_{n}:=\Lambda\left[V+W_{n}\right] \geqslant \Lambda[\mathcal{R}]=\lim _{n \rightarrow \infty} \Lambda_{n}$. By Theorem 1 , for all $\phi \in L^{2}\left(\mathbb{R}^{3}, \mathbb{C}^{2}\right)$ and any $\lambda \in\left(-1, \Lambda_{n}\right]$, we have that

$4^{\mathrm{e}}$ SÉRIE - TOME $40-2007-\mathrm{N}^{\circ} 6$ 


$$
\begin{aligned}
\int_{\mathbb{R}^{3}} V|\phi|^{2} d x+\int_{\mathbb{R}^{3}} W_{n}|\phi|^{2} d x & \leqslant \int_{\mathbb{R}^{3}} \frac{|\sigma \cdot \nabla \phi|^{2}}{1+\lambda+V+W_{n}} d x+(1-\lambda) \int_{\mathbb{R}^{3}}|\phi|^{2} d x \\
& \leqslant \int_{\mathbb{R}^{3}} \frac{|\sigma \cdot \nabla \phi|^{2}}{1+\lambda+V} d x+(1-\lambda) \int_{\mathbb{R}^{3}}|\phi|^{2} d x
\end{aligned}
$$

since $W_{n} \geqslant 0$. Passing to the limit in the above inequality proves (8) for all $\lambda \in(-1, \Lambda[\mathcal{R}]]$. Indeed, for every $\phi$ such that the right-hand side of (8) is finite, we have

$$
\lim _{n \rightarrow+\infty} \int_{\mathbb{R}^{3}} \frac{|\sigma \cdot \nabla \phi|^{2}}{1+\lambda+V+W_{n}} d x=\int_{\mathbb{R}^{3}} \frac{|\sigma \cdot \nabla \phi|^{2}}{1+\lambda+V} d x,
$$

since $\lambda \geqslant 0$ and $\mathcal{S}=\lim _{n \rightarrow \infty} W_{n}$ is supported in a 0 measure set of $\mathbb{R}^{3}$.

\section{Acknowledgements}

This work has been partially supported by European Programs HPRN-CT \# 2002-00277 \& 00282, and by the project ACCQUAREL of the French National Research Agency (ANR). Second author supported by the grant MTM2005-08430 of MEC (Spain) and FEDER. Fourth author supported by the grant MTM 2004-03029 of MEC (Spain) and FEDER.

\section{REFERENCES}

[1] Dolbeault J., Esteban M.J., Loss M., Vega L., An analytical proof of Hardy-like inequalities related to the Dirac operator, J. Funct. Anal. 216 (2004) 1-21.

[2] Dolbeault J., Esteban M.J., SÉRÉ E., On the eigenvalues of operators with gaps. Application to Dirac operators, J. Funct. Anal. 174 (2000) 208-226.

[3] Dolbeault J., Esteban M.J., SÉRÉ E., General results on the eigenvalues of operators with gaps, arising from both ends of the gaps. Application to Dirac operators, J. Eur. Math. Soc. 8 (2006) 243251.

[4] Duondikoetxea J., Vega L., Some weighted Gagliardo-Nirenberg inequalities and applications, Proc. Amer. Math. Soc. 135 (2007) 2795-2802.

[5] Filippas S., Tertikas A., Optimizing improved Hardy inequalities, J. Funct. Anal. 192 (2002) 186233.

[6] Talman J.D., Minimax principle for the Dirac equation, Phys. Rev. Lett. 57 (1986) 1091-1094.

[7] Thaller B., The Dirac Equation, Texts and Monographs in Physics, Springer-Verlag, Berlin, 1992.

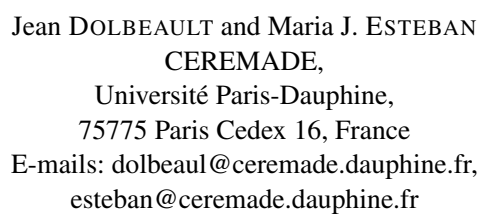


Javier DUOANDIKOETXEA and Luis VEGA

Universidad del País Vasco,

Departamento de Matemáticas, Apartado 644,

48080 Bilbao, Spain

E-mails: javier.duoandikoetxea@ehu.es, luis.vega@ehu.es 